\title{
Time-Fractal in Living Objects
}

\author{
Andras Szasz \\ Department of Biotechnics, Szent Istvan University, Budaörs, Hungary \\ Email: biotech@gek.szie.hu
}

How to cite this paper: Szasz, A. (2022) Time-Fractal in Living Objects. Open Journal of Biophysics, 12, 1-26.

https://doi.org/10.4236/ojbiphy.2022.121001

Received: October 3, 2021

Accepted: December 11, 2021

Published: December 14, 2021

Copyright (c) 2022 by author(s) and Scientific Research Publishing Inc. This work is licensed under the Creative Commons Attribution International License (CC BY 4.0).

http://creativecommons.org/licenses/by/4.0/

\begin{abstract}
Homeostasis creates self-organized synchrony of the body's reactions, and despite the energetically open system with intensive external and internal interactions, it is robustly stable. Importantly the self-organized system has scaling behaviors in its allometry, internal structures, and dynamic processes. The system works stochastically. Deterministic reductionism has validity only by the great average of the probabilistic processes. The system's dynamics have a characteristic distribution of signals, which may be characterized by their frequency distribution, creating a particular "noise" $1 / f$ of the power density. The stochastic processes produce resonances pumped by various noise spectra. The chemical processes are mostly driven by enzymatic processes, which also have noise-dependent resonant optimizing. The resonance frequencies are as many as many enzymatic reactions exist in the target.
\end{abstract}

\section{Keywords}

Homeostasis, Self-Organizing, Feedbacks, Complexity, Resonance, Stochastic Processes, 1/f Noise, Dissipation, Enzymatic Reactions

\section{Introduction}

All parts of the biosystems are energetically open. The micro and macro environment have a decisional influence on their processes. The system exchanges energy and information with its environment. According to a well-defined balance, the processes are dynamic and interconnected with each other, the homeostasis [1]. This dynamic stability is self-organized [2], and despite the intensive interactions, it is robustly stable at large order of magnitudes [3]. The dynamic stability is regulated and controlled by the homeostatic feedback mechanisms [4], keeping the balance between promoters and suppressors in the complete system [5]. The living network is undoubtedly not a simple addition of its parts [6]. It forms a complex structure [7]. Theoretical biology faces a severe 
challenge of complexity [8].

Regardless of its living of lifeless state build forms, the natural structures are far from the possibility to describe them in the frame of Euclidean geometry with straight lines and circles. The natural structures are self-organized and mostly form fractal structures [9]. The fractal geometry in life makes it possible to categorize the living species by their allometric comparison [10] comparison of complex morphogenetic differences [11]. This type of universality of the complex feedback mechanisms controls the dynamic equilibrium maintaining the homeostasis [12]. Fractal models represent an excellent approach to explaining the living processes' structural development [13], even for the genetic code structure [14].

The genetic code construction uses Kronecker products (KP) of matrixes with binary numbers. The construction of KP sequences the same template and so represents fractals too [15] [16]. The generated nucleotide sequences characteristic of various living systems form a fractal pattern. An extension of KP construction introduces blocks and a multifractal approach [17], which fits the living complexity [18].

The fractal description is suitable for extending the dynamic physiological processes and analyzing the fractal properties in time [19]. The time-fractal studies are based on the research of the structure of various signals [20] [21]. The dynamism of the energetically open living systems dominantly involves self-organizing processes allowing their fractal description [22]. The time fractals reflect the complex space-time approach developed a new discipline, fractal physiology [23] [24], expressing the collectivity of the processes [25].

The modulation of the external bioelectromagnetic signals has well-explained principles. The carrier frequency helps in the selection mechanisms, while its modulation supports homeostasis by its time fractal $(1 / f)$ frequency distribution [26]. The modulation could have multiple effects locally and systematically. The local force for the homeostatic control acts as a further selection factor regarding the lost control of the tumorous cells. Furthermore, the modulation forces the healthy dynamical order providing a compulsory process for apoptosis of the out-of-control cells. $H R V$ may characterize the homeostasis [27], presenting the complexity of the system.

The well applied time-fractal current flow may activate the structural fractals in the living systems, and the personal fractal structure could modify the time-fractal pattern, too [28]. The fundamentally nonlinear physiological system dynamics work on the edge of chaos, a border of order and disorder showing a constant dynamic interplay between these states [29]. The challenge of the homeostatic equilibrium is the apparent chaos. The chaos looks complete randomness only. However, the chaos in biosystems results from the stochastic self-organizing and the energetically open system, which directly and permanently interacts with the environment. Its structural and temporal structure is fractal, which appears in the fundamental arrangements of the self-similar building and dynamism of the 
energy exchanges internally and externally. The living processes are complex. They are in self-organized criticality (SOC) [30], which is formulated, as the "life at the edge of chaos" [31]. This chaos is the realization of a well-organized stochastic (probabilistic) system [32]. The chaos is only an ostensible complete disorder [33].

\section{Methods}

\subsection{Fluctuations}

An organism has a finite number of possible states. These states could be characterized in terms of operational quality utilizing a diagnostic parameter (signals). All signals have an average in time, and the signals fluctuate around this value in a controlled band. The random fluctuation sets various states (microstates) of the body, which exist only briefly and appear as fluctuation. The temporal fluctuation is regarded as a noise of the signal. The noise of living processes usually does not fluctuate randomly. The homeostatic control of the body regulates them. The minimal number of diagnostic signals is defined by the quasi-independent, weakly overlapping regulation intervals. The number of these quasi-independent diagnostic signals does not change during the system meets the conditions of the healthy dynamical equilibrium, the homeostasis. The average values, the fluctuation band, and the distribution of the frequencies may vary, depending on age and adaptation to changing environmental conditions. These quantities are called macroscopic diagnostic determinants and the status vector with $D_{i}$ diagnostic states:

$$
D_{i}=D_{i}(\boldsymbol{X}, \boldsymbol{Y}) \quad(i=1,2, \cdots, n)
$$

where $\boldsymbol{X}$ and $\boldsymbol{Y}$ are the signals of the system and outside environment, respectively. Due to the short time realized microstates, the number of diagnostic states is significantly less than the numberof its determinant signals $D_{i}$, consequently, the microstates appear as statistical statements. The same homeostatic macrostate has a wide variety of microstates that change rapidly over time, fluctuating around the averages. The probability that the microstate falls in the interval $(\boldsymbol{X}, \boldsymbol{X}+\mathrm{d} \boldsymbol{X})$ at time $t$, i.e., the probability density $w(\boldsymbol{X}, t)$ with:

$$
P_{\boldsymbol{X}}(\boldsymbol{X}<\boldsymbol{\xi} \leq \boldsymbol{X}+\mathrm{d} \boldsymbol{X})=w(\boldsymbol{X}, t) \mathrm{d} X
$$

Consequently $D_{i}$ is given by $w(\boldsymbol{X}, t)$ it is a stochastic determinant which primarily we characterize with its average (mean value)

$$
\left\langle D_{i}\right\rangle=\int_{(X)} D_{i}(\boldsymbol{X}, \boldsymbol{Y}) w(X, t) \mathrm{d} X \quad(i=1,2, \cdots, n)
$$

and its variance

$$
\sigma_{D_{i}}=\left\langle\left(D_{i}-\left\langle D_{i}\right\rangle\right)^{2}\right\rangle \quad(i=1,2, \cdots, n)
$$

where \langle\rangle denotes the average of the values. The failure of the dynamic equilibrium when $\left|D_{i}-\left\langle D_{i}\right\rangle\right|$ is larger than a predetermined threshold with a limiting value $\left(l_{D_{i}}\right)$. According to the Chebyshev theorem [34] the probability that 
$\left|D_{i}-\left\langle D_{i}\right\rangle\right|>l_{D_{i}}$ (so the system is out from the healthy homeostasis) is:

$$
P_{\text {fail }}\left(\left|D_{i}-\left\langle D_{i}\right\rangle\right|>l_{D_{i}}\right) \leq \frac{\sigma_{D_{i}}^{2}}{l_{D_{i}}^{2}}=\frac{\left(D_{i}-\left\langle D_{i}\right\rangle\right)^{2}}{l_{D_{i}}^{2}}
$$

In a healthy state the $P_{\text {fail }}$ is small. The $\left\langle D_{i}\right\rangle$ average characterizes this state. The conventional diagnostics controls $\left\langle D_{i}\right\rangle$ values only, regarding the patient healthy when the fluctuations $f_{D_{i}}=D_{i}-\left\langle D_{i}\right\rangle$ remain within a tolerance band $l_{D_{i}}$. However, the fluctuation carries essential information about the microstates. Changes in the regulative processes could drastically modify the fluctuation of the signal without changing its average value. Study the noise spectrum may predict modifications of the regulative feedbacks, so it has diagnostic value.

The living, dynamic equilibrium is well-regulated but in a probabilistic way. The time-dependent processes realize the observed signal with a probability, as the actual exposition from the possibilities of the fluctuations of the measured signal.

The vital principle is the feedback mechanism, which controls the balance within a predetermined range around the reference value. It is usually well modeled with fuzzy logic, an approach to counting "degrees of truth" rather than the usual "true or false" decisions [35]. This logic governs homeostatic equilibria in all ranges of space and time in living systems. This uncertain value is undoubtedly in a controlled reference interval, were strongly interconnected negative feedback loops regulate the balance in the micro and macro ranges, forming the system's dynamic stability.

These phenomena request a stochastic approach (probability of events dependent on time) instead of conventional thinking based on deterministic changes [36]. Deterministic reductionism can mislead the research. The homeostasis is often ignored and used as a static framework for effects [37]. The stochastic approach is fundamental in biological dynamism [38]. The dynamic homeostatic equilibrium keeps the system in a stable but constantly changing state.

\subsection{Stochastic and Deterministic Approach}

A model calculation of tumor growth shows the strength of the stochastic approach. In a simple example, the growth of a tumor can be described deterministically. The deterministic change of tumor mass $\left(\Delta M_{t}\right)$ by observation time $(\Delta t)$ is proportional with its actual mass $\left(M_{t}\right)$ :

$$
\Delta M_{t}(t)=k M_{t}(t) \Delta t
$$

where $k$ is a constant. A well-known exponential solution uses the mass of the tumor at the start of its observation $\left(M_{0}\right)$ :

$$
\frac{\mathrm{d} M_{t}(t)}{\mathrm{d} t}=k M_{t}(t) \Rightarrow M_{t}(t)=M_{0} \mathrm{e}^{k t}
$$

In a deterministic way, the prognostic task of oncology would be simple regarding exponential growth. However, the process is stochastic, requesting the 
step-by-step analysis of the development of the tumor. We follow the additional or disappearing individual cells producing the mass growth. The probability $P_{M_{t}}$ to add a cell to the tumor at $t$ time during $\Delta t$ interval is proportional with $k M_{t}(t) \Delta t$, as we assumed initially been in (6). Then the probability equation with the added and eliminated cells in time interval $\Delta t$ is:

$$
P_{M_{t}}(t+\Delta t)=P_{M_{t}}(t)+k\left(M_{t}-1\right) \Delta t P_{M_{t}-1}(t)-k M_{t} \Delta t P_{M_{t}}(t)
$$

It depends on the added cells to the tumor from the previous time interval $\left(P_{M_{t}}(t+\Delta t)\right)$ and the eliminated cells in the actual time $\left(-k M_{t} \Delta t P_{M_{t}}(t)\right)$ considering the process in one step before $\left(k\left(M_{t}-1\right) \Delta t P_{M_{t}-1}(t)\right)$. In a differential equation form:

$$
\frac{\mathrm{d} P_{M_{t}}(t)}{\mathrm{d} t}=k\left(M_{t}-1\right) P_{M_{t}-1}(t)-k M_{t} P_{M_{t}}
$$

When we start from a single cell $\left(P_{M_{t}}(0)=1\right.$ if $M_{0}=1$, and $P_{M_{t}}(0)=0$ in every other case), the solution of (9) at $M_{t} \geq M_{0}$ cases:

$$
P_{M_{t}}(t)=\left(\begin{array}{c}
M_{t}-1 \\
M_{t}-M_{0}
\end{array}\right) \mathrm{e}^{-k M_{0} t}\left(1-\mathrm{e}^{-k t}\right)^{M_{t}-M_{0}}
$$

Compare (7) and (10) how they are different! The deterministic approach (7) is continuous in time, running in real values, while the stochastic, probability-based approach (10) jumps on integers, building up the tumor-mass step by step. The deterministic equation gives a fixed result, while the stochastic shows "only" probability. It is interesting to see that the deterministic result is the particular case of the stochastic one, the deterministic $P_{M_{t}}(t)=\left\langle M_{t}(t)\right\rangle$ condition does not depend of the actual number of steps. Consequently, the averaging of the stochastic probability results provide the deterministic solution:

$$
P_{M_{t}}(t)=\left\langle M_{t}(t)\right\rangle=\sum_{M_{t}=M_{0}}^{\infty} M_{t} P_{M_{t}}(t)=M_{0} \mathrm{e}^{k t}
$$

\subsection{The Fluctuation Phenomena}

The signals follow the living, dynamic interactions, the molecular changes, and the chemical and physical excitations give a structured noise. The power spectral density of a signal $(S(f))$, is the power of the noise (fluctuation) per unit of bandwidth. Define the work of the $x(t)$ stochastic process:

$$
W:=\int_{-\infty}^{\infty} x^{2}(t) \mathrm{d} t
$$

The (12) with the Parseval's formula may be evaluated

$$
W=\int_{-\infty}^{\infty} x^{2}(t) \mathrm{d} t=\int_{-\infty}^{\infty} S(f) \mathrm{d} f
$$

where $S(f)$ is the spectral power density in any random stationary case. The Fourier transform of $x(t)$ stochastic process is the primary step to study the phenomena [39],

$$
X(f)=\frac{1}{\sqrt{2 \pi}} \int_{-\infty}^{\infty} x(t) \mathrm{e}^{-j 2 \pi f t} \mathrm{~d} t:=F\{x(t)\}
$$


where the spectral density function $S(f)$ is:

$$
S(f)=\frac{|X(f)|^{2}}{2 \pi}
$$

The even function of the frequency, i.e., $S(f)=S(-f)$.

The $S(f)$ gives the intensity of noise as a function of spatial frequency, measured in $\frac{W}{H z}=J$, characterizing the stochastic signal with the $f$ frequency. The most straightforward complex noise follows normal (Gaussian) distribution (the amplitudes have normal distribution), and its power function $S(f)$ is self-similar through many orders of magnitudes. In this simple case, the $S(f)$ :

$$
S(f)=\frac{A}{f^{\alpha}}
$$

The $\alpha$ exponent in (16) formally refers to optics, noted as the "color" of the noise. The white-noise is flat ( $\alpha=0$ ), the pink-noise has $\alpha=1$, and other colors are described by various other numbers up to $\alpha=2$, the brown-noise. So, the $S(f)$ of pink-noise inversely depends on $f$ frequency, noted as $1 / f$ noise. The $1 / f$ noise carries the self-similar structure of living processes having a time-fractal covering the life's dynamism [40] [41]. The dynamical fractal structure of living systems marks the self-organizing both in geometric and time structures and dynamically regulates the living matter [42], defines time-fractal structure in stochastic way of the living systems [43], a $1 /$ fluctuation. The physiological control shows $1 / f$ spectrum [44]. One of the most studied such spectra is the heart rate variability (HRV).

This $1 / f$ noise has a particular behavior. Each octave interval (halving or doubling in frequency) carries an equal amount of noise energy. The living system makes special signal processing due to its self-organized symmetry, so it transforms the white noise to pink [45], forming the most common signal in biological systems [46].

Stochastic signals additionally to $S(f)$ are usually characterized by their autocorrelation function $R_{X X}\left(t_{1}, t_{2}\right)$. The autocorrelation measures how the signal correlates with a delayed copy of itself in the function of time-lag $\left(\tau=t_{2}-t_{1}\right)$, measuring the signal in $t_{1}$ and subsequent $t_{2}$ in $X$ position. The autocorrelation evaluation is a mathematical tool for finding repeating patterns, looking for periodicity in the signal. It allows identifying the existence of the biological chain processes. The $S(f)$ and $R_{X X}\left(t_{1}, t_{2}\right)$ functions are not independent, they could be converted to each other by Fourier transformation. Measuring the power density $S(f)$ of a signal is easier than its autocorrelation, so usually the studies concentrate on the power density function.

\section{Results}

\subsection{White Noise}

All frequencies in the entire interval have the same $A$ amplitude in the white 
noise spectrum:

$$
S(f)=A \propto \frac{1}{f^{\alpha}}
$$

i.e., from (16), $\alpha=0$. Consequently, the autocorrelation function is completely uncorrelated:

$$
\begin{aligned}
R_{X X}(\tau) & =\frac{1}{2 \pi} \int_{-\infty}^{\infty} S(f) \cos (2 \pi f \tau) \mathrm{d} \pi f=\int_{0}^{\infty} S(f) \cos (2 \pi f \tau) \mathrm{d} f \\
& =\frac{A}{2} \int_{0}^{\infty} 2 \cos (2 \pi f \tau) \mathrm{d} f=\frac{A}{2} \delta(\tau)
\end{aligned}
$$

The band constraint in a limited interval, up to $f_{\max }$ upper-frequency limit affects a longer-term correlation:

$$
\begin{aligned}
R_{X X}(\tau) & =\frac{1}{2 \pi} \int_{-\omega_{0}}^{\omega_{0}} S(f) \cos (2 \pi f \tau) \mathrm{d} \pi f=\int_{0}^{f_{\max }} S(f) \cos (2 \pi f \tau) \mathrm{d} f \\
& =\frac{A}{2} \int_{0}^{f_{\max }} 2 \cos (2 \pi f \tau) \mathrm{d} f=A \frac{\sin \left(2 \pi f_{\max } \tau\right)}{2 \pi f_{\max } \tau}
\end{aligned}
$$

For example, the completely flat $S(f)$ limited to the frequency-band [-10 10] has well-defined autocorrelation Figure 1:

The correlation function oscillates, so the correlation length does not monotonically decrease in band-limited white noise.

\subsection{The $1 / f$ Noise}

A stationary random process has an indefinite duration. To introduce a modified density spectrum, consider a finite segment of the random process $x(t)$ of duration $2 T$, defined by:

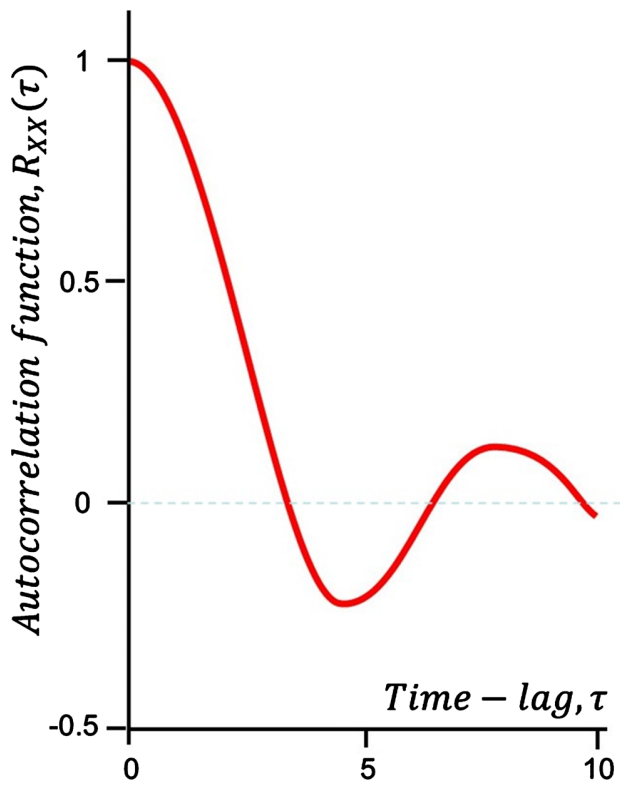

Figure 1. The correlation function $R_{X X}(\tau)$ of band-limited white noise than the $S(f)=f^{0}=\cos t$. 


$$
x_{T}=\left\{\begin{array}{ll}
x(t), & -T \leq t \leq T \\
0, & \text { otherwise }
\end{array} \text { and } \lim _{T \rightarrow \infty} x_{T}(t)=x(t)\right.
$$

According to (14), the Fourier transform of $x_{T}(t)$ has the form of

$$
X(f, T)=\frac{1}{\sqrt{2 \pi}} \int_{-T}^{T} x_{T}(t) \mathrm{e}^{-j 2 \pi f t} \mathrm{~d} t
$$

The Fourier transform of the function $x(a \cdot t)$, where $a$ is an arbitrary complex number, and $f$ is the frequency:

$$
F\{x(a \cdot t)\}=\frac{1}{a} X\left(\frac{f}{a}\right)
$$

Use (21) and (22) we get:

$$
F\left\{x_{T}(a t)\right\}=\frac{1}{a} X\left(\frac{f}{a}, T\right)
$$

Using Parseval's formula and (15):

$$
\lim _{T \rightarrow \infty} \frac{1}{2 T} \int_{-T}^{T} x^{2}(t) \mathrm{d} t=\int_{-\infty}^{\infty} S(f) \mathrm{d} f \quad\left\{S(f)=\frac{1}{2 \pi} \lim _{T \rightarrow \infty} \frac{|X(f, T)|^{2}}{2 T}\right\}
$$

The living processes are basically self-similar, so it is convenient to define the self-similarity of a stochastic process. A stochastic process is said to be self-similar if the effective power of the stochastic process representation $x(t)$ equals the effective power of the representation $x(a t)$ defined over time scale [at], for every a positive scalar, i.e.:

$$
\lim _{T \rightarrow \infty} \frac{1}{2 T} \int_{-T}^{T} x^{2}(t) \mathrm{d} t=\lim _{T \rightarrow \infty} \frac{1}{2 T} \int_{-T}^{T} x^{2}(a t) \mathrm{d}(a t)
$$

And so from (22) and (20), we get

$$
a \int_{-\infty}^{\infty} \frac{1}{a^{2}} S\left(\frac{f}{a}\right) \mathrm{d} f=\int_{-\infty}^{\infty} S(f) \mathrm{d} f
$$

Also, for the power spectral density function, the functional equation may be expressed:

$$
S\left(\frac{f}{a}\right)=a S(f)
$$

for every positive scalar $a$ and every scalar $f$. To solve this equation, we assume that $f>0$ and set for $a$ the value $a=f$ :

$$
S(f)=\frac{S(1)}{f}
$$

On the other hand, if $f<0$ then $f=-|f|$, and

$$
\frac{1}{a} S\left(\frac{f}{a}\right)=\frac{1}{a} S\left(-\frac{|f|}{a}\right)=S(f)
$$

Let us set for $a$ the value $a=|f|$ and take into account that the power density function is even, so we obtain the $1 / f$ spectrum, or "pink-noise": 


$$
S(f)=\frac{S(1)}{|f|}
$$

The autocorrelation function of $S(f) \propto \frac{1}{f^{1}}$ pink noise with Fourier transformation has a singular result:

$$
\begin{aligned}
R_{X X}(\tau) & =\frac{1}{2 \pi} \int_{-\infty}^{\infty} S(f) \cos (2 \pi f \tau) \mathrm{d} \pi f \\
& =\int_{0}^{\infty} \frac{1}{f} \cos (2 \pi f \tau) \mathrm{d} f \\
& =\int_{0}^{\infty} \frac{\cos (2 \pi f \tau)}{2 \pi f \tau} \mathrm{d}(2 \pi f \tau)
\end{aligned}
$$

follows the $C i(x)$ function:

$$
\operatorname{Ci}(x)=-\int_{x}^{\infty} \frac{\cos (2 \pi f \tau)}{2 \pi f \tau} \frac{\cos x^{\prime}}{x^{\prime}} \mathrm{d} x^{\prime}
$$

Due to $\operatorname{Ci}(\infty)=0$, the autocorrelation of $1 / f$ noise in long time-lag is zero Figure 2 .

By the ergodic hypothesis [47], the autocorrelation function of a stationary random process $x(t)$ can be defined as

$$
R_{x x}(\tau)=\lim _{T \rightarrow \infty} \frac{1}{2 T} \int_{-T}^{T} x(t) x(t+\tau) \mathrm{d} t \quad\left\{R_{x x}(\tau)=R_{x x}(-\tau)\right\}
$$

where $\tau$ is the time-lag. The relation between autocorrelation function and the power density spectrum can be expressed by the Fourier transform of the autocorrelation function (Wiener-Khinchine theorem), namely:

$$
\begin{aligned}
& R_{x x}(f)=\frac{1}{\sqrt{2 \pi}} \int_{-\infty}^{\infty} R_{x x}(\tau) \mathrm{e}^{-j 2 \pi f \tau} \mathrm{d} \tau \\
& R_{x x}(\tau)=\frac{1}{\sqrt{2 \pi}} \int_{-\infty}^{\infty} R_{x x}(f) \mathrm{e}^{j 2 \pi f \tau} \mathrm{d} f
\end{aligned}
$$

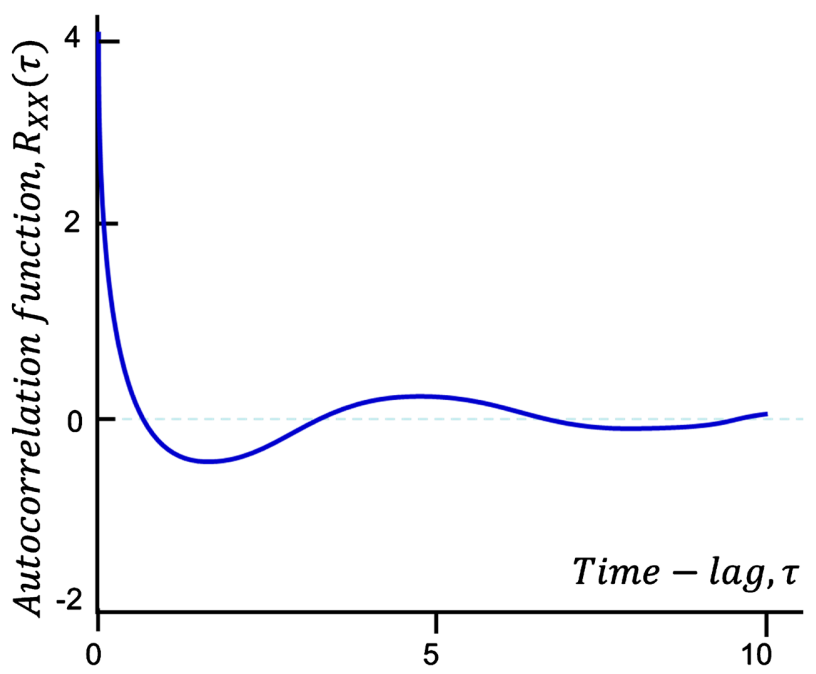

Figure 2. Autocorrelation function of $1 /$ f noise (negative integral cosine function, $-\mathrm{Ci}(x))$. 
From these (considering [36] and [48]), we may conclude

$$
R_{x x}(\tau)=\int_{-\infty}^{\infty} S(f) \mathrm{e}^{j 2 \pi f \tau} \mathrm{d} f=\int_{-\infty}^{\infty} \frac{S(1)}{|f|} \mathrm{e}^{j 2 \pi f \tau} \mathrm{d} f=\frac{\sqrt{2 \pi} S(1)}{|\tau|}
$$

Assuming the lower cutoff frequency $f_{\min }$, the function of such an approximate $1 / f$ noise correlation from (31)

$$
\phi(\tau)=\int_{f_{\min }}^{\infty} \frac{1}{f} \cos (2 \pi f \tau) \mathrm{d} f=\int_{f_{\min }}^{\infty} \frac{\cos (2 \pi f \tau)}{2 \pi f \tau} \mathrm{d}(2 \pi f \tau)=-C i\left(2 \pi f_{\min } \tau\right)
$$

The procedure is also shown in Figure 2.

It can be seen from the figure that here too, there is a problem with the introduction of the correlation length since the correlation function oscillates.

In the case where the lower cutoff frequency is minimal, the argument of the $C i$-function is small even at significant offset times. Then the correlation function is as shown in Figure 3.

It appears that this case can be approximated by the sum of white noise and a virtually constant correlation function. More precisely, the can be asymptotically approximated by

$$
\varphi(\tau)=-\left(\gamma+\ln \left(2 \pi f_{0} \tau\right)\right)
$$

with a function where $\gamma \cong 5772$ is the Euler-Mascheroni constant.

The autocorrelation function of $1 / f^{\alpha} \quad(\alpha \neq 0$ and $\alpha \neq 1)$ :

$$
R_{X X}(\tau)=\int_{0}^{\infty} \frac{1}{f^{\alpha}} \cos (2 \pi f \tau) \mathrm{d} f=\frac{1}{\tau^{1-\alpha}} \frac{\sqrt{\pi}}{\sqrt{2} \sin \left(\frac{\alpha \pi}{2}\right) \Gamma(1-\alpha)}
$$

Note that colored noises do not fit the white and pink noises, so the basic noises have no common expression.

The pink noise cannot be described with the classical apparatus of nonequilibrium thermodynamics. Macroscopic fluctuation characterizes the

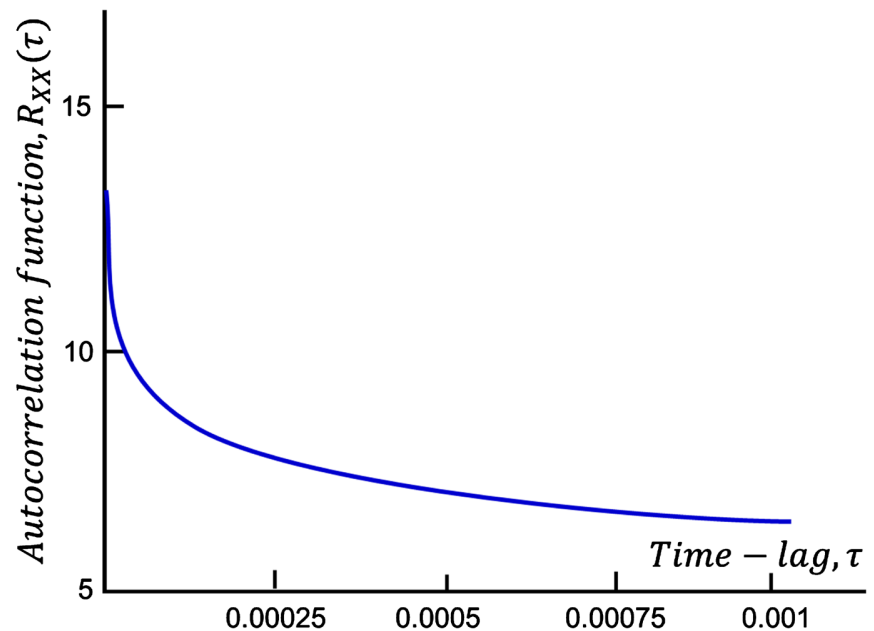

Figure 3. Autocorrelation function $1 /$ fnoise for very low cutoff frequency (negative, integral cosine function, $-\mathrm{Ci}(x))$. 
thermodynamic processes. The range of space in which the fluctuation occurs is not uniform concerning the fluctuating quantity $(s)$ but is thermodynamically in equilibrium at all points. The latter means that the exchange of extensive amounts characteristic of fluctuation between spatial domains during the relaxation period of equilibrium is negligible. A further feature of thermodynamic fluctuations is that the fluctuation persists for a finite time and that the rate of change of each $a_{i}(i=1,2, \cdots, n)$ extensive can be expressed in terms of the extensive amounts involved in the fluctuation, i.e.

$$
\frac{\mathrm{d} a_{i}}{\mathrm{~d} t}=f\left(a_{1}, a_{2}, \cdots, a_{n}\right) \quad(i=1,2, \cdots, n)
$$

Let be an extensive one whose relaxation time is much longer than the others. Then the fluctuation can be described by this single extensive one. When (39) is linear and returns to the equilibrium position of the system, then the equation is a one-sided fluctuation process, completely deterministic, with no noise in it:

$$
\frac{\mathrm{d} a}{\mathrm{~d} t}=-\lambda a
$$

Solving (41):

$$
a(t)=a(0) \mathrm{e}^{-\lambda t}
$$

Then the correlation function is:

$$
R_{a a}(\tau)=\langle a(\tau) a(0)\rangle=[a(0)]^{2} \mathrm{e}^{-\lambda|\tau|}
$$

and its power spectrum:

$$
S(i \omega)=\int_{-\infty}^{\infty} R_{a a}(\tau) \mathrm{e}^{-i \omega \tau} \mathrm{d} \tau=[a(0)]^{2} \frac{\lambda}{\lambda^{2}+\omega^{2}}
$$

For stochasticity, the necessary noise appears in the fluctuation and spectrum for the whole, but the considerations lead to (43) are deterministic. Therefore, it is assumed that this deterministic signal is repeated randomly, forming a noise of a series of randomly repeated deterministic signals. Introducing a white noise function into the deterministic equation (like is in the Langevin equation) applies the amplitudes of the white noise spectrum that corresponds to the noise spectrum given by the deterministic random fluctuation and accordingly with the correlation function too. This is white noise $\left(\frac{1}{\omega}\right)$ for small $\omega$ values, while Brown noise $\left(\frac{1}{\omega^{2}}\right)$ for large values.

In the case of pink-noise, these considerations do not work. The Fourier transform connects the $S(f)$ power function and the $R_{x x}$ autocirreklation function:

$$
S(i \omega) \Leftrightarrow R_{a a}(\tau) \Rightarrow \frac{1}{|b|} S\left(\frac{i \omega}{b}\right) \Leftrightarrow R_{a a}(b \tau)
$$

Because 


$$
S(i \omega)=\frac{1}{|\omega|}
$$

because of this

$$
\frac{1}{|b|} S\left(\frac{i \omega}{b}\right)=\frac{1}{|\omega|}
$$

so it follows that

$$
R_{a a}(\tau)=R_{a a}(b \tau)
$$

The correlation function is constant in this case, so the pink noise correlated in the same way for each shift, so there can be no thermodynamic fluctuation!

Starting with such randomized deterministic fluctuations, we get equivalents to form of (40), like:

$$
\frac{\mathrm{d} a}{\mathrm{~d} t}=-\lambda a=-\frac{1}{\tau} a
$$

In this case, instead of (41), we get the following spectrum:

$$
S(i \omega)=\int_{-\infty}^{\infty} f_{a a}(\tau) \mathrm{e}^{-i \omega \tau} \mathrm{d} \tau=[a(0)]^{2} \frac{\tau}{1+(\tau \omega)^{2}}
$$

Assuming that the temporal correlation length probability density function is lognormal, the resulting noise spectrum is: $1 / f^{\alpha}$. It is the same as the originally white-noise pumped stochastic case. It is confusing, of course, that this process started from deterministic distribution, but it was overcome by assuming that there is a random series of such deterministic fluctuations.

Two stochastic processes can be considered equivalent if their noise spectrum is the same. Based on this, we introduce a stochastic excitation term $q(t)$ to (48):

$$
\frac{\mathrm{d} a}{\mathrm{~d} t}=-\frac{1}{\tau} a+q(t)
$$

The $q(t)$ spectrum is chosen of the signal resulting from the solution of the equation is equal to the power spectrum of the fluctuation (49). This can always be done. To prove this, Fourier transforms Equation (50), then we get that

$$
\left(i \omega+\frac{1}{\tau}\right) a=q(\omega) \rightarrow a(\omega)=\frac{\tau}{1+(i \omega \tau)} q(\omega)
$$

Hence the power spectrum

$$
S(\omega)=\frac{\tau^{2}}{1+(\omega \tau)^{2}}|q(\omega)|^{2}
$$

The following choice leads to the desired result:

$$
q(\omega)=\frac{a(0)}{\sqrt{\tau}}
$$

Consequently, if $q(t)$ is a white noise with $\frac{a(0)}{\sqrt{\tau}}$ amplitude, then the noise 
spectrum of the signal is the same as the noise spectrum of the fluctuation.

\subsection{Orstein-Uhlenbeck Process}

The power spectrum of a random series of such deterministic fluctuations differs from the white-noise pumped Langevin solution only in a proportionality factor. We approach the fluctuation by decomposing it into the sum of quasi-periodic stochastic processes of different statistically independent time scales. The quasi-periodic stochastic processes with different time scales also have different frequency scales. All such component processes are assumed to be statistically similar. Note the increase of a stochastic $X(t)$ process $X(t+\mathrm{d} t)-X(t)$ without memory with $\Theta$-function:

$$
X(t+\mathrm{d} t)-X(t)=\Theta[X(t), t, \mathrm{~d} t] .
$$

Assume that $\Theta[X(t), t, \mathrm{~d} t]$ is a smooth function of the $X, t, \mathrm{~d} t$ variables and that $X(t)$ is continuous:

$$
\lim _{\mathrm{d} t \rightarrow 0} X(t+\mathrm{d} t)=X(t) .
$$

The approach that the observed noise by the emission of subsequent processchains in statistical mechanics, the Markov process [49] describes the chain reaction, which is used in biology too [50]. The Markovian recursive successive building the $X(t+\mathrm{d} t)$, while the function $X(t)$ from where it was derived depends only from $t$ in memory-less construction, using:

$$
\begin{aligned}
\Theta[X(t), t, \mathrm{~d} t] & =\sum_{i=1}^{n} X\left(t+i \frac{\mathrm{d} t}{n}\right)-X\left(t+(i-1) \frac{\mathrm{d} t}{n}\right) \\
& =\sum_{i=1}^{n} \Theta\left[X\left(t+(i-1) \frac{\mathrm{d} t}{n}\right), t+(i-1) \frac{\mathrm{d} t}{n}, \frac{\mathrm{d} t}{n}\right]
\end{aligned}
$$

Since $\mathrm{d} t$ can be chosen to be arbitrarily small, the $t_{i-1}=t+(i-1) \frac{\mathrm{d} t}{n}$ can be placed in any proximity of the $t$ times by choosing $n$ large enough. Exploiting the continuity, in this case:

$$
\begin{aligned}
& t_{i-1} \rightarrow t, \quad X\left(t_{i-1}\right)=X(t) \\
& \Theta[X(t), t, \mathrm{~d} t]=\sum_{i=1}^{n} \Theta_{i}\left[X(t), t, \frac{\mathrm{d} t}{n}\right]
\end{aligned}
$$

Here, the $\Theta_{i}\left[X(t), t, \frac{\mathrm{d} t}{n}\right]$ terms can be considered as representations of the $\Theta\left[X(t), t, \frac{\mathrm{d} t}{n}\right]$ variable that is statistically independent due to being the memory free of the process. Since $n$ is arbitrarily large, it follows from the central limit theorem that $\Theta[X(t), t, \mathrm{~d} t]$ is the sum of $n$ statistically independent $\Theta_{i}\left[X(t), t, \frac{\mathrm{d} t}{n}\right]$ probability variables. Hence, this probability variable distributes normally. The following properties follow from the property of normally distributed random variables: 


$$
\begin{aligned}
& \langle\Theta[X(t), t, \mathrm{~d} t]\rangle=n \cdot\left\langle\Theta\left[X(t), t, \frac{\mathrm{d} t}{n}\right]\right\rangle \\
& \langle\langle\Theta[X(t), t, \mathrm{~d} t]\rangle\rangle=n \cdot\left\langle\left\langle\Theta\left[X(t), t, \frac{\mathrm{d} t}{n}\right]\right\rangle\right\rangle
\end{aligned}
$$

where \langle\rangle notes the mean, and $\langle\langle\rangle\rangle$ is the standard deviation. Solving function equations

$$
\begin{aligned}
& \langle\Theta[X(t), t, \mathrm{~d} t]\rangle=A[X(t), t] \mathrm{d} t \\
& \langle\langle\Theta[X(t), t, \mathrm{~d} t]\rangle\rangle=D[X(t), t] \mathrm{d} t
\end{aligned}
$$

where $A$ and $D$ are smooth functions of $X$ and $t$, and $D>0$. Considering the normality of (55) and (60):

$$
\begin{aligned}
X(t+\mathrm{d} t)-X(t) & =\Theta[X(t), t, \mathrm{~d} t] \\
& =N[A(X, t) \mathrm{d} t, D(X, t) \mathrm{d} t] \\
& =A(X, t) \mathrm{d} t+D^{\frac{1}{2}} N(0,1) \mathrm{d} t^{\frac{1}{2}}
\end{aligned}
$$

where $N(0,1)$ is the unit standard deviation squared normal distribution stochastic process with zero means. Turning to a differential equation, we get the following nonlinear generalized Langevin equation

$$
\frac{\mathrm{d} X}{\mathrm{~d} t}=A(X, t)+D^{\frac{1}{2}}(X, t) \Gamma(t)
$$

driven by normally distributed white noise:

$$
\Gamma(t)=\lim _{\mathrm{d} t \rightarrow 0} N\left(0, \mathrm{~d} t^{-1}\right)
$$

In the Gillespie sense [51], the stochastic process is self-similar, resolved to a sum of statistically independent terms normally distributed within the studied interval. Consider the simplest of the self-similar stochastic processes in (61):

$$
\frac{\mathrm{d} X}{\mathrm{~d} t}=-\frac{1}{\tau} X+D^{\frac{1}{2}} \Gamma(t)
$$

where $\tau$ is the time constant of the process.

The describes an Ornstein-Uhlenbeck process (OUP), which is stochastic and follows a normal (Gaussian) distribution. The OUP is homogeneous in time. Its homogeneity in time allows the OUP to describe it simply with the stochastic interaction of an energy source and the connected energy-consuming system Figure 4, allowing linear transformations of space and time variables [52].

The central value is exponentially decreasing, and a white noise drives it. The exponential decay should be uniformly distributed rather than lognormal, the maximum entropy belongs to $1 / f$, and then the equation and distribution of the distribution should lead to $1 / f$.

If we use a lognormal distribution in the interval [53], modifying (63) by $D=\frac{D_{0}}{\sqrt{\tau}}$ [54]: 


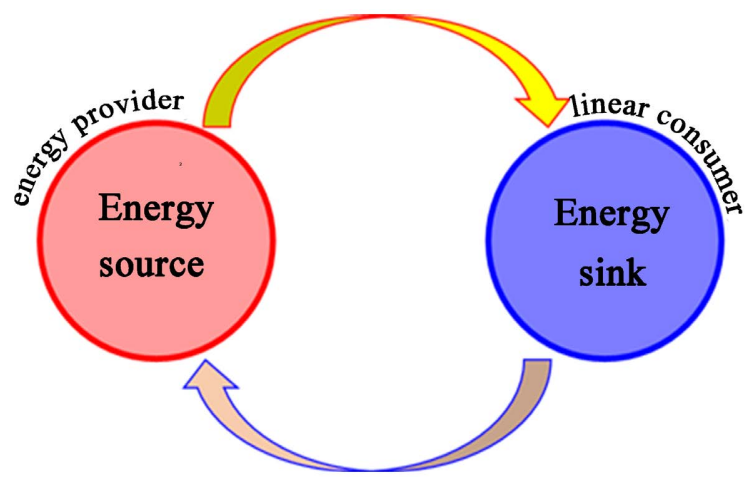

Figure 4. The simplest relation of the energy source (reservoir, mechanical, electronic, etc.) and the linear consumer (energy-sink mechanical electronic, etc.)

$$
\frac{\mathrm{d} X}{\mathrm{~d} t}=-\frac{1}{\tau} X+\frac{D_{0}^{\frac{1}{2}}}{\sqrt{\tau}} \Gamma(t)
$$

Thus, the power spectrum of this is distributed by the lognormal of the time domain, asymptotically $1 / \tau$. The equation describes the noise of a system excited by white noise consisting of an energy store (e.g., mass, rotating mass, capacitor, inductance) and a linear attenuation (e.g., fluid resistance, ohmic resistance).The power spectrum of the process:

$$
S(\omega, \tau)=\frac{D_{0} \tau_{s}^{2}}{1+\left(\omega \tau_{s}\right)^{2}}
$$

Here $\tau_{s}$ is the time constant of the system, which can also be considered the natural time scale of the stochastic process. Let's define

$$
\lambda=\frac{1}{\tau_{s}}
$$

a frequency scale at which we want to characterize stochastic processes. Let $G(\lambda) \mathrm{d} \lambda$ a be the number of stochastic processes in the frequency interval $(\lambda, \lambda+\mathrm{d} \lambda)$, then the energy spectrum of the stochastic processes in the interval between the frequency scales $\left(\lambda_{2}, \lambda_{1}\right)$ :

$$
S\left(\omega, \lambda_{1}, \lambda_{2}\right)=\int_{\lambda_{1}}^{\lambda_{2}} \frac{D \cdot G(\lambda)}{\lambda^{2}+\omega^{2}} \mathrm{~d} \lambda
$$

If the distribution is uniform, that is, if,

$$
G(\lambda) \mathrm{d} \lambda=\frac{\mathrm{d} \lambda}{\lambda_{2}-\lambda_{1}}
$$

then we get that

$$
S\left(f, \lambda_{1}, \lambda_{2}\right)=\int_{\lambda_{1}}^{\lambda_{2}} \frac{D \cdot G(\lambda)}{\lambda^{2}+\omega^{2}} \mathrm{~d} \lambda= \begin{cases}D & \text { if } 0<\omega \ll \lambda_{1} \ll \lambda_{2} \\ \frac{D \pi}{2 \omega\left(\lambda_{2}-\lambda_{1}\right)} & \text { if } \lambda_{1} \ll \omega \ll \lambda_{2} \\ \frac{D}{\omega^{2}} & \text { if } \lambda_{1} \ll \lambda_{2} \ll \omega\end{cases}
$$


a well-known result gives white noise in the first interval, pink in the second, and brown (Wiener noise) in the third.

When the relaxation rate is uniform in an interval $\left[f_{1}, f_{2}\right]$ and the applied amplitude doesn't change. Hence the spectrum of OUP, $S(f)=\frac{1}{f^{\alpha}}$ has three well distinguishable frequency parts Figure 5.

\subsection{Importance of the Self-Similarity}

The $\tau_{s}$ the time constant of the system in (65) generates the stochastic signal. The $\tau_{s}$ can be considered as the natural time scale of the stochastic process that characterizes the two-point correlation function of the stochastic process. Indeed, the two-point correlation function from (65) shows the degree of correlation decreases exponentially with $\tau$ time constant:

$$
\phi_{X X}(\vartheta)=F^{-1}\left[S\left(\omega, \tau_{s}\right)\right]=F^{-1}\left[\frac{D_{0} \tau_{s}}{1+\left(\omega \tau_{s}\right)^{2}}\right]=D_{0} \mathrm{e}^{-\frac{\vartheta}{\tau_{s}}}
$$

This feature of $\tau_{s}$ is the temporal correlation length.

The complexity of the system involves a $G\left(\tau_{s}\right) \mathrm{d} \tau_{s}$ number of statistically independent stochastic processes in the temporal correlation length interval $\left(\tau_{s}, \tau_{s}+\mathrm{d} \tau_{s}\right)$, then the resulting energy spectrum of the stochastic processes in the $(0, \infty)$ interval is:

$$
S(\omega)=\int_{0}^{\infty} \frac{D_{0} \tau_{s} G\left(\tau_{s}\right)}{1+\left(\tau_{s} \omega\right)^{2}} \mathrm{~d} \tau_{s}
$$

when the distribution is scale variant, i.e.:

$$
G\left(\tau_{s}\right) \mathrm{d} \tau_{s}=\frac{\mathrm{d} \tau_{s}}{\tau_{s}}
$$

form, then using Equation (70) a

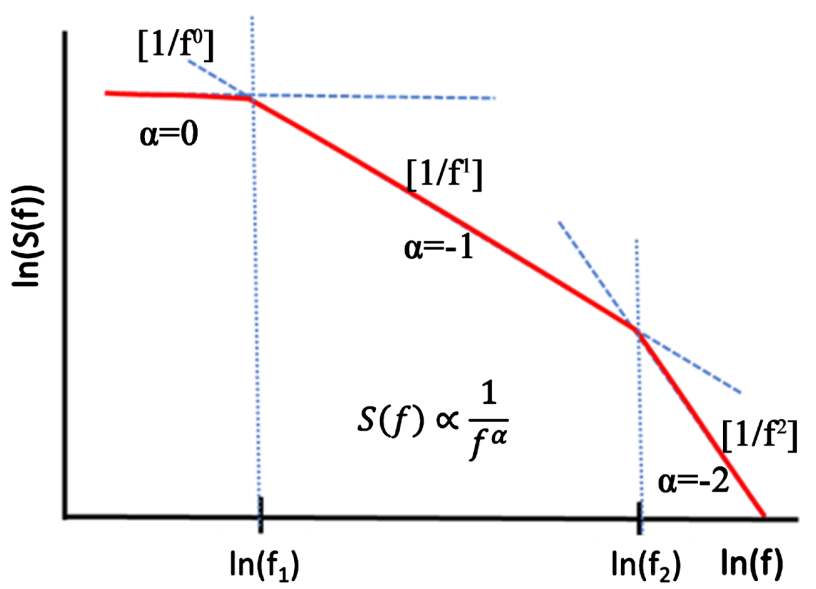

Figure 5. The power density function is divided into three distinguishable parts in Ornstein-Uhlenbeck process. The $\left\{f_{1}, f_{2}\right\}$ interval, when the probability of realization of the $f$ frequencies are equal. 


$$
\int_{0}^{\infty} \frac{1}{1+\left(\tau_{s} \omega\right)^{2}} \mathrm{~d} \tau_{s}=\frac{\pi}{2} \frac{1}{\omega}
$$

improper integrated, we get the desired result:

$$
S(\omega)=\int_{0}^{\infty} \frac{D_{0} \tau_{s} G\left(\tau_{s}\right)}{1+\left(\tau_{s} \omega\right)^{2}} \mathrm{~d} \tau_{s}=D_{0} \int_{0}^{\infty} \frac{\tau_{s} \frac{1}{\tau_{s}}}{1+\left(\tau_{s} \omega\right)^{2}} \mathrm{~d} \tau=\frac{D_{0} \pi}{2} \frac{1}{\omega} \propto \frac{1}{f}
$$

The scale invariance means that the probability scale is independent,

$$
G\left(\tau_{s}\right) \mathrm{d} \tau_{s}=G\left(\alpha \tau_{s}\right) \mathrm{d} \alpha \tau_{s} \Rightarrow \frac{\mathrm{d} \alpha \tau_{s}}{\alpha \tau_{s}}=\frac{\mathrm{d} \tau_{s}}{\tau_{s}}
$$

In the case where only self-similarity is required, e.g., as a function of density. That is

$$
G\left(\alpha \tau_{s}\right)=\alpha^{\beta} G\left(\tau_{s}\right)
$$

then we get that

$$
G\left(\tau_{s}\right)=\tau_{s}^{\beta}
$$

In this case $\beta=-1$, it provides $1 / f$ noise. If we require only self-similarity, we get from (71) and (77) that the noise spectrum of signals in the interval $(0, \infty)$ is:

$$
S(\omega)=\int_{0}^{\infty} \frac{D_{0} \tau_{s} G\left(\tau_{s}\right)}{1+\left(\tau_{s} \omega\right)^{2}} \mathrm{~d} \tau_{s}=\int_{0}^{\infty} \frac{D_{0} \tau_{s}^{\beta+1}}{1+\left(\tau_{s} \omega\right)^{2}} \mathrm{~d} \tau_{s}
$$

Due to the physical image, the integrated a

$$
S(\omega)=\int_{0}^{\infty} \frac{D_{0} \tau_{s}^{\beta+1}}{1+\left(\tau_{s} \omega\right)^{2}} \mathrm{~d} \tau_{s}=\frac{D_{0}}{\omega^{\beta+2}} \int_{0}^{\infty} \frac{\left(\omega \tau_{s}\right)^{\beta+1}}{1+\left(\tau_{s} \omega\right)^{2}} \mathrm{~d}\left(\omega \tau_{s}\right)
$$

to shape.

The integral is generally unpredictable. Fortunately, in the case of interest to us, if $0<\beta<2$ the impropriety integral can be given in the closed-form:

$$
\int_{0}^{\infty} \frac{\left(\omega \tau_{s}\right)^{\beta+1}}{1+\left(\tau_{s} \omega\right)^{2}} \mathrm{~d}\left(\omega \tau_{s}\right)=\frac{\pi}{2 \sin \left(\frac{(\beta+2) \pi}{2}\right)}=A
$$

This gives (79) that

$$
S(\omega)=\frac{D_{0}}{\omega^{\beta+2}} \int_{0}^{\infty} \frac{\left(\omega \tau_{s}\right)^{\beta+1}}{1+\left(\tau_{s} \omega\right)^{2}} \mathrm{~d}\left(\omega \tau_{s}\right)=\frac{D_{0} A}{\omega^{\beta+2}}
$$

The self-similar distribution function is thus the condition a shaped power spectrum:

$$
S(\omega) \propto \frac{1}{\omega^{\alpha}}
$$

The above considerations can be generalized to a large extent. 
Namely, if instead of $D=\frac{D_{0}}{\sqrt{\tau}}$ in (64) use

$$
D=\frac{D_{0}}{\tau^{\gamma}}
$$

We start from the stochastic process described by the equation, using normally distributed white noise as before in (62). Then the power spectrum will be:

$$
S(\omega, \tau)=\frac{D_{0} \tau^{2-\gamma}}{1+(\omega \tau)^{2}}
$$

If we require only self-similarity, we get from (84) and (59) the noise spectrum of signals in the interval $(0, \infty)$ :

$$
S(\omega)=\int_{0}^{\infty} \frac{D_{0} \tau^{2-\gamma} G(\tau)}{1+(\tau \omega)^{2}} \mathrm{~d} \tau=\int_{0}^{\infty} \frac{D_{0} \tau^{\beta-\gamma+2}}{1+(\tau \omega)^{2}} \mathrm{~d} \tau
$$

Due to the physical image, the integral is arranged into a form:

$$
S(\omega)=\int_{0}^{\infty} \frac{D_{0} \tau^{\beta-\gamma+2}}{1+(\tau \omega)^{2}} \mathrm{~d} \tau=\frac{D_{0}}{\omega^{\beta-\gamma+3}} \int_{0}^{\infty} \frac{(\omega \tau)^{\beta-\gamma+2}}{1+(\tau \omega)^{2}} \mathrm{~d}(\omega \tau)
$$

In the case of interest to us, if the $0<\beta-\gamma+3<2$ the impropriety integral can be given again in closed form:

$$
\int_{0}^{\infty} \frac{(\omega \tau)^{\beta-\gamma+2}}{1+(\tau \omega)^{2}} d(\omega \tau)=\frac{\pi}{2 \sin \left(\frac{(\beta-\gamma+3) \pi}{2}\right)}=A
$$

which gives from (78):

$$
S(\omega)=\frac{D_{0}}{\omega^{\beta-\gamma+3}} \int_{0}^{\infty} \frac{(\omega \tau)^{\beta-\gamma+2}}{1+(\tau \omega)^{2}} \mathrm{~d}(\omega \tau)=\frac{D_{0} A}{\omega^{\beta-\gamma+3}}
$$

The self-similarity is again desired the power spectrum:

$$
S(\omega) \propto \frac{1}{\omega^{\alpha}}
$$

This result concludes to an important note: the self-similarity is a more fundamental feature of the noise than its $1 / f$ shape. Support this we derive instead of the $1 / f^{\alpha}$ the noise spectrum from thermodynamic fluctuations, [55].

\subsection{Energy Dissipation}

Considering that the quantum theory of the dissipative systems is not adequately worked out, we stay within the range of the classical theory. We suppose that the pieces of information necessary for the communication are carried by the analog signals describing the physicochemical state of the individual cells. Furthermore, we are going to suppose that the self-similar Markov processes can represent the state of coaching biological subsystems. Gillespie could show that from this assumption, the equation describing the dynamics of processes can be concluded. This is the generalized Langevin equation [56]: 


$$
\frac{\mathrm{d} X_{i}}{\mathrm{~d} t}=A_{i}\left(X_{j}, t\right)+D_{i}^{\frac{1}{2}}\left(X_{j}, t\right) \Gamma(t),(i=0,1,2, \cdots, N-1)
$$

where

$$
\Gamma(t)=\lim _{\mathrm{d} t \rightarrow 0} N\left(0, \mathrm{~d} t^{-1}\right)
$$

is the white-noise with zero mean value, infinite dispersion, and normal distribution. Let us decompose the $A_{i}\left(X_{j}, t\right)$ function into three parts:

$$
A_{i}\left(X_{j}, t\right)=f_{i}(t)+A_{i}\left(X_{i}\right)+\sum_{k=0}^{N-1} c_{i k} X_{k}
$$

where the $c_{i k}$ elements form a cyclic matrix.

$$
\bar{C}=\left[\begin{array}{cccc}
c_{0} & c_{1} & \cdots & c_{N-1} \\
c_{N-1} & c_{0} & \cdots & c_{N-2} \\
\vdots & \vdots & \ddots & \vdots \\
c_{1} & c_{2} & \cdots & c_{0}
\end{array}\right]
$$

$A_{i}\left(X_{i}\right)$ can be nonlinear and the $f_{i}(t)$ is the time function generated by the internal active processes of the cell. It is reasonable to assume that $A_{i}\left(X_{i}\right)$ is identical for each cell, and at the same way, we may suppose that $D_{i}$ is constant for each cell. This latter can be justified because each cellis to be found in the same heat conditions. We did not assumed any confinement for the $f_{i}(t)$ function. The proposed equation isthe generalization of the model of the coupled damped oscillators, which showed [57] that the stochastic resonance is included in the forms of motion. We are going to examine a case where the social signal has low amplitude; therefore, the nonlinear members can be neglected. Then (91):

$$
\frac{\mathrm{d} X_{i}}{\mathrm{~d} t}=f_{i}(t)+\sum_{k=0}^{N-1} c_{i k} X_{k}+D^{\frac{1}{2}} \Psi(t),(i=0,2, \cdots, N-1)
$$

\subsection{Cellular Communication in a Noisy Environment}

The effective field strength of thermal noise was first calculated by Weaver and Astumian [58]. The Weaver \& Astumian model (W-A model) assumed changes in the field strength result from fluctuations of space charges on both sides of the cellular membrane and further showed a thermal noise limit at low frequencies. Kaune [59] revisited the W-A model and showed that the field strengths typical of thermal noise converge to zero at low frequencies. Therefore, the $\mathrm{W}$-A model does not describe this region appropriately. However, thermal noise in Kaune's model [19] is assumed to be synchronized (coherent) over the entire cell membrane. This assumption is called the coherence condition. Unfortunately, thermal noise is unlikely to be coherent over a large structure such as a cell. Therefore, the calculation that followed is limited to a highly unlikely special case. Kaune set all noise-generators to be equipotential based on the coherence condition by assuming parallel connectivity and the equivalent electrical circuit. As the coherence condition does not hold in the general case, the equipotential assumption also does not hold in the general case. We generalized the problem 
and developed a solution [60]. Our results proved when there are only zero-mode currents present. The limit does not exist. However, at non-zero currents, the thermal noise does limit the efficacy of electromagnetic effects in low frequencies. The zero mode is the action by central symmetry for all individual cells instead of the translation symmetry of the usually applied outside field effects.

The topological construction is an essential factor of the cellular organization, [61], irrespective it is alive or not. The cellular structure, because of some topological reasons, develops preferring special coordination arrangements [62] and could arrange a self-organized collectivity [63] [64]. It was discovered that the division tendency is very low in the cell population, small in number [65]. For the start of a significant cell division, a critical cell density is necessary. This was later observed on a self-synchronization of chemical oscillators [66]. The topological importance was assumed in living cellular cultures also, [67], declaring that not the cell density but the position (coordination number) of cells related to each other determines what is favorable or not favorable from the point of view of division. This hypothesis was later justified experimentally [68].

The cells in developed multicellular living objects are grouped into organs to perform certain tasks in a network together. This network extends inside the cells and has suitable connection points outside the cell wall, ensuring with this to involve the cellular mechanisms in the tasks of the network. The cytoskeleton of the cells provides the basic cellular information-transfers intracellularly. The internal cytoskeleton network has transmembrane bridges (e.g., adherent connections, junctions) connecting the matrix structure on the outer side of the cell through the polar protein molecules [69]. The network develops by polymerization [70], where the water structures of aqueous electrolyte arrange the extracellular matrix partially. For example, the formed "intercellular filaments" in epithelial tissues implements the mechanical coupling of individual cells [71] [72]. Ordered water creates efficient proton conduction mechanisms [73] that disordered water does not have. The hydrogen bridges transport the protons, which is crucial in living systems [74]. This high-speed and low dissipation of the transport propagation is based on Grotthuss-mechanism [75].

The healthy cells are under the control of others in the network ("social" signaling [76], a collective action). Social information should spread within the body without loss of information. However, the environment is noisy, and the living information exchange faces this challenge. Now, we are going to prove that among the modes belonging to the eigenvectors of the matrix (93) of equation (91), there are modes of zero noise spectrum. It is well known that any cyclic matrix can be diagonalized by the transformation matrix [77], that is

$$
T=\frac{1}{\sqrt{N}}\left[\begin{array}{cccccc}
1 & 1 & \cdots & 1 & \cdots & 1 \\
1 & a & \cdots & a^{i} & \cdots & a^{N-1} \\
1 & a^{j} & \cdots & a^{j i} & \cdots & a^{j(N-1)} \\
\vdots & \vdots & \ddots & \vdots & \ddots & \vdots \\
1 & a^{N-1} & \cdots & a^{(N-1) i} & \cdots & a^{(N-1)^{2}}
\end{array}\right],
$$


where $a=\mathrm{e}^{i 2 \pi / N}$. Applying this transformation to the Equation (94), we obtain:

$$
\frac{\mathrm{d} x_{s i}}{\mathrm{~d} t}=\lambda_{\mathrm{i}} x_{s i}+f_{s i}(t)+\Gamma_{s i}(t)(i=0, \cdots, N-1) .
$$

Here the new coordinates and the eigenvalues of the cyclic matrix are

$$
\begin{aligned}
& x_{s i}=\frac{1}{\sqrt{N}} \sum_{k=0}^{N-1} a^{-i k} x_{k}^{\prime}, \quad \Gamma_{s i}(t)=D^{\frac{1}{2}} \frac{1}{\sqrt{N}} \sum_{k=0}^{N-1} a^{-i k} \Gamma(t), \\
& \lambda_{j}=\sum_{k=0}^{N-1} a^{j k} c_{k}, \quad f_{s i}(t)=\frac{1}{\sqrt{N}} \sum_{k=0}^{N-1} a^{-i k} f_{i}(t), \quad(j=0, \cdots, N-1)
\end{aligned}
$$

Let us consider any one of the new

$$
\Gamma_{s i}(t)=D^{\frac{1}{2}} \frac{1}{\sqrt{N}} \sum_{k=0}^{N-1} a^{-i k} \Gamma(t)
$$

noise components for which $k \neq 0$ (non-zero order component). Let us take the Fourier to transform thereof and consider that the amplitudes are unitary in the white-noise spectrum. Then we get that

$$
\Gamma_{s i}(t)=D^{\frac{1}{2}} \frac{1}{\sqrt{N}} \sum_{k=0}^{N-1} a^{-i k}, k \neq 0
$$

On the other hand, we know that

$$
\sum_{k=0}^{N-1} a^{-i k}=0
$$

In consequence, every non-zero order mode is noiseless because:

$$
\Gamma_{s i}(t)=0, k \neq 0
$$

So the zero-order noises are not only limitless by thermal noises, but the signal exchange in such a way is noiseless.

\section{Conclusion}

The stochastic processes drive the homeostatic harmony, synchronizes the processes by environmental noises, while the system performs the important internal signal communications noiselessly. The dynamic stochastic living systems involve characteristic resonances. Particular resonant frequencies differentiate and describe the various enzymatic processes.

\section{Acknowledgements}

This work was supported by the Hungarian National Research Development and Innovation Office PIACI KFI grant: 2019-1.1.1-PIACI-KFI-2019-00011.

\section{Conflicts of Interest}

The author declares no conflicts of interest regarding the publication of this paper.

\section{References}

[1] Modell, H., Cliff, W., Michael, J., et al. (2015) A Physiologist's View of Homeostasis. 
Advances in Physiology Education, 39, 259-266.

https://doi.org/10.1152/advan.00107.2015

[2] Walleczek, J. (2000) Self-Organized Biological Dynamics \& Nonlinear Control. Cambridge Univ. Press, Cambridge. https://doi.org/10.1017/CBO9780511535338

[3] Anteneodo, C. and da Luz, M.G.E. (2010) Complex Dynamics of Life at Different Scales: From Genomic to Global Environmental Issues. Philosophical Transactions of the Royal Society A, 368, 5561-5568. https://doi.org/10.1098/rsta.2010.0286

[4] Turrigiano, G. (2007) Homeostatic Signaling: The Positive Side of Negative Feedback. Current Opinion in Neurobiology, 17, 318-324. https://doi.org/10.1016/j.conb.2007.04.004

[5] Lloyd, D., Aon, M.A. and Cortassa, S. (2001) Why Homeodynamics, Not Homeostasis? The Scientific World, 1, 133-145. https://doi.org/10.1100/tsw.2001.20

[6] Mohr, H. (1977) Structure and Significance of Science. Springer, New York, 102.

[7] Theise, N.D. and Kafatos, M.C. (2013) Complementarity in Biological Systems-A Complexity View. Periodicals, 18, 11-20. https://doi.org/10.1002/cplx.21453

[8] Seel, M. and Ladik, J. (2019) Chapter 1. The Tragicomedy of Modern Theoretical Biology. In: Advances in Quantum Chemistry, Elsevier, Amsterdam, Vol. 81, 1-13. https://doi.org/10.1016/bs.aiq.2019.11.001

[9] Mandelbrot, B.B. (1977) The Fractal Geometry of Nature. Times Books, New York.

[10] Camazine, S., Deneubourg, J.L., Franks, N.R., et al. (2003) Self-Organization in Biological Systems. Princeton Studies in Complexity. Princeton Univ. Press, Princeton.

[11] Losa, G.A. (2009) The Fractal Geometry of Life. Rivista di Biologia, 102, 29-59.

[12] Losa, G.A. (2012) Fractals and Their Contribution to Biology and Medicine. Medicographia, 34, 365-374.

[13] Weibel, E.R. (1991). Fractal Geometry: A Design Principle for Living Organisms. American Journal of Physiology, 261, L361-L369. https://doi.org/10.1152/ajplung.1991.261.6.L361

[14] Petoukhov, S.V. (2008) The Degeneracy of the Genetic Code and Hadamard Matrices. https://arxiv.org/ftp/arxiv/papers/0802/0802.3366.pdf

[15] Voevudko, A.E. (2018) Fractal Dimension of the Kronecker Product. Mathematics.

[16] Voevudko, A.E. (2017) Generating Kronecker Product Based Fractals. CodeProject. https://www.codeproject.com/Articles/1189288/Generating-Kronecker-Product-Bas ed-Fractals

[17] Moreno, S., Robles-Granda, P. and Neville, J. (2013) Block Kronecker Product Graph Model.

https://www.semanticscholar.org/paper/Block-Kronecker-Product-Graph-Model-M oreno-Robles-Granda/ad35b967418cd01f2899b507ae008b816b4b1d82

[18] Leskovec, J., Chakrabarti, D., Kleinber, J., Faloutsos, C., et al. (2010) Kronecker Graphs: An Approach to Modeling Networks. Journal of Machine Learning Research, 11, 985-1042.

[19] Roca, J.L. (2018) Fractal-Based Techniques for Physiological Time Series: An Updated Approach. De Gruyter, Berlin.

[20] Vrobel, S. (2011) Fractal Time, Studies of Nonlinear Phenomena in Life Science. Vol. 14, World Scientific, Singapore. https://doi.org/10.1142/7659

[21] Wornell, G.W. (1996) Signal Processing with Fractals, a Wavelet-Based Approach. Prentice Hall Signal Processing Series, Prentice Hall, Upper Saddle River. 
[22] Sturmberg, J. and West, B.J. (2013) Fractals in Physiology and Medicine. In: Sturmberg, J. and Martin, C., Eds., Handbook of Systems and Complexity in Health, Springer, Berlin, 171-192. https://doi.org/10.1007/978-1-4614-4998-0

[23] Deering, W. and West, B.J. (1992) Fractal Physiology. IEEE Engineering in Medicine and Biology, 11, 40-46. https://doi.org/10.1109/51.139035

[24] Bassingthwaighte, J.B., Leibovitch, L.S. and West, B.J. (1994) Fractal Physiology. Oxford Univ. Press, New York. https://doi.org/10.1007/978-1-4614-7572-9

[25] Goldenfeld, N. and Woese, C. (2010) Life Is Physics: Evolution as a Collective Phenomenon Far from Equilibrium.

[26] Szasz, A. and Szasz, O. (2020) Ch. 17. Time-Fractal Modulation of Modulated Electro-Hyperthermia (mEHT). In: Szasz, A., Ed., Challenges and Solutions of Oncological Hyperthermia, Cambridge Scholars, Newcastle upon Tyne, 377-415.

[27] Scheff, J.D., Griffel, B., Corbett, S.A., Calvano, S.E. and Androulakis, I.A. (2014) On Heart Rate Variability and Autonomic Activity in Homeostasis and in Systemic Inflammation. Mathematical Biosciences, 252, 36-44.

https://doi.org/10.1016/j.mbs.2014.03.010

[28] Goldberger, A.L., Bhargava, V., West, B.J. and Mandell, A.J. (1985) On a Mechanism of Cardiac Electrical Stability-The Fractal Hypothesis. Biophysics Journal, 48, 525-528. https://doi.org/10.1016/S0006-3495(85)83808-X

[29] Kauffman, S.A. and Johnsen, S. (1991) Coevolution to the Edge of Chaos: Coupled Fitness Landscapes, Poised States, and Coevolutionary Avalanches. Journal of Theoretical Biology, 149, 467-505. https://doi.org/10.1016/S0022-5193(05)80094-3

[30] Bak, P., Tang, C. and Wiesenfeld, K. (1988) Self-Organized Criticality. Physical Review $A$, 38, 364-374. https://doi.org/10.1103/PhysRevA.38.364

[31] Lewin, R. (1992) Complexity, Life at the Edge of Chaos. University of Chicago Press, Chicago.

[32] Ito, K. and Gunji, Y.P. (1994) Self-Organisation of Living Systems towards Criticality at the Edge of Chaos. Biosystems, 33, 17-24.

https://doi.org/10.1016/0303-2647(94)90057-4

[33] Prigogine, I. and Stengers, I. (1985) Order out of Chaos. Flamingo, London. https://doi.org/10.1063/1.2813716

[34] Chernick, M.R. (2011) The Essentials of Biostatistics for Physicians, Nurses, and Clinicians. John Wiley \& Sons, Hoboken, 49-50.

https://doi.org/10.1002/9781118071953

[35] Wierman, M.J. (2010) An Introduction to Mathematics of Uncertainty. Hoors Program.

http://typo3.creighton.edu/fileadmin/user/CCAS/programs/fuzzy_math/docs/MOU .pdf

[36] Eskov, V.M., Filatova, O.E., Eskov, V.V., et al. (2017) The Evolution of the Idea of Homeostasis: Determinism, Stochastics, and Chaos-Self-Organization. Biophysics, 62, 809-820. https://doi.org/10.1134/S0006350917050074

[37] Billman, G.E. (2020) Homeostasis: The Underappreciated and Far Too Often Ignored Central Organizing Principle of Physiology. Frontiers in Physiology, 11, 200. https://doi.org/10.3389/fphys.2020.00200

[38] Mode, C.J., Durrett, R., Klebaner, F., et al. (2013) Applications of Stochastic Processes in Biology and Medicine. International Journal of Stochastic Analysis, 2013, Article ID: 790625. https://doi.org/10.1155/2013/790625

[39] Nigam, N.C. (1983) Introduction to Random Vibrations. The MIT Press, Cambridge. 
[40] Cramer, F. (1995) Chaos and Order (The Complex Structure of Living Systems). $\mathrm{VCH}$, Weinheim.

[41] Peng, C.K., Buldyrev, S.V., Hausdorff, J.M., et al. (1994) Fractals in Biology and Medicine: From DNA to the Heartbeat. In: Bunde, A. and Havlin, S., Eds., Fractals in Science, Springer-Verlag, Berlin, 49-87.

https://doi.org/10.1007/978-3-662-11777-4_3

[42] Musha, T. and Sawada, Y. (1994) Physics of the Living State. IOS Press, Amsterdam.

[43] Wentian, L. (1989) Spatial 1/f Spectra in Open Dynamical Systems. Europhysics Letters, 10, 395-400. https://doi.org/10.1209/0295-5075/10/5/001

[44] Kim, J.J., Parker, S., Henderson, T. and Kirby, J.N. (2020) Physiological Fractals: Visual and Statistical Evidence across Timescales and Experimental States. Journal of the Royal Society Interface, 17, Article ID: 20200334. https://doi.org/10.1098/rsif.2020.0334

[45] Szendro, P., Vincze, G. and Szasz, A. (2001) Bio-Response on White-Noise Excitation. Electromagnetic Biology and Medicine, 20, 215-229.

https://doi.org/10.1081/JBC-100104145

[46] Szendro, P., Vincze, G. and Szasz, A. (2001) Pink-Noise Behaviour of Biosystems. European Biophysics Journal, 30, 227-231. https://doi.org/10.1007/s002490100143

[47] Szasz, D. (1994) Boltzmann's Ergodic Hypothesis, a Conjecture for Centuries? The International Symposium in Honour of Boltzmann's 150 th Birthday, Vienna, 24-26 February 1994, 1-23.

[48] Sneddon, I. (1955) Handbuch der Physik Bd. II. Springer Verlag, Berlin.

[49] Seneta, E. (2016) Markov Chains as Models in Statistical Mechanics. Statistical Science, 31, 399-414. https://doi.org/10.1214/16-STS568

[50] Tsong, T.Y. and Chang, C.-H. (2007) A Markovian Engine for a Biological Energy Transducer: A Catalitic Wheel. Biosystems, 88, 323-333. https://doi.org/10.1016/j.biosystems.2006.08.014

[51] Gillespie, D.T. (1977) Exact Stochastic Simulation of Coupled Chemical Reactions. The Journal of Physical Chemistry, 81, 2340-2361. https://doi.org/10.1021/j100540a008

[52] Doob, J.L. (1942) The Brownian Movement and Stochastic Equations. Annals of Mathematics, 43, 351-369. https://doi.org/10.2307/1968873

[53] Schlesinger, M.S. (1987) Fractal Time and 1/f Noise in Complex Systems. Annals of the New York Academy of Sciences, 504, 214-228. https://doi.org/10.1111/j.1749-6632.1987.tb48734.x

[54] Shesinger, M. and West, B.J. (1988) Versus Noise. In: Stanly, H.E. and Ostrowsky, N., Eds., Random Fluctuations and Pattern Growth. Experiments and Models, Kluwer Academic Publishers, Dordrecht, 320-324.

[55] Milotti, E. (2002) 1/f Noise: A Pedagogical Review. Classical Physics. https://arxiv.org/abs/physics/0204033

[56] Gillespie, D.T. (1992) Markov Processes. Academic Press, San Diego.

[57] White, D.C. and Woodson, H.H. (1959) Electromechanical Energy Conversion. John Wiley and Sons, Inc., New York.

[58] Weaver, J.C. and Astumian, R.D. (1990) The Response of Living Cells to Very Week Electric Fields: The Thermal Noise Limit. Science, 247, 459-462.

https://doi.org/10.1126/science.2300806

[59] Kaune, W.T. (2002) Thermal Noises Limit on the Sensitivity of Cellular Membranes 
to Power Frequency Electric and Magnetic Fields. Bioelectromagnetics, 23, 622-628. https://doi.org/10.1002/bem.10060

[60] Vincze, G., Szász, A. and Szasz, N. (2005) On the Thermal Noise Limit of Cellular Membranes. Bioelectromagnetics, 26, 28-35. https://doi.org/10.1002/bem.20051

[61] Zsoldos, I., Szendro, P., Watson, L., et al. (2001) Topological Correlation in Amorphous Structures. Computational Materials Science, 20, 28-36. https://doi.org/10.1016/S0927-0256(00)00120-8

[62] Vincze, G., Zsoldos, I. and Szasz, A. (2004) On the Aboav-Weaire Law. Journal of Geometry and Physics, 51, 1-12. https://doi.org/10.1016/j.geomphys.2003.08.003

[63] Zsoldos, I. and Szasz, A. (1999) Appearance of Collectivity in Two-Dimensional Cellular Structures. Computational Materials Science, 15, 441-448. https://doi.org/10.1016/S0927-0256(99)00031-2

[64] Maryan, M.I., Kikineshi, A.A. and Szasz, A. (2001) Self-Organizing Processes and Dissipative Structure Formation in the Non-Crystalline Materials. Physics and Chemie Status Solidi, 2, 585-593.

[65] Puck, T.T., Marcus, P.I. and Cieciura, S.J. (1956) Clonal Growth of Mammalian Cells in Vitro: Growth Characteristics of Colonies from Single HeLa Cells with and without a "Feeder" Layer. Journal of Experimental Medicine, 103, 273-283. https://doi.org/10.1084/jem.103.2.273

[66] Taylor, A.F., Tinsley, M.R., Wang, F., et al. (2009) Dynamical Quorum Sensing and Synchronization in Large Populations of Chemical Oscillators. Science, 323, 614-617. https://doi.org/10.1126/science.1166253

[67] Caer, G.L. (1991) Topological Models of Cellular Structures. Journal of Physics A: Mathematical and General, 24, 1307-1317. https://doi.org/10.1088/0305-4470/24/6/022

[68] Puck, T.T. and Marcus, P.I. (1955) A Rapid Method for Viable Cell Titration and Clone Production with HeLa Cells in Tissue Culture: The Use of X-Irradiated Cells to Supply Conditioning Factors. Proceedings of the National Academy of Sciences of the United States of America, 41, 432-437. https://doi.org/10.1073/pnas.41.7.432

[69] Hamerof, S.R. (1988) Coherence in the Cytoskeleton: Implications for Biological Information Processing. In: Froelich, H., Ed., Biological Coherence and Response to External Stimuli, Springer Verlag, Berlin, 242-265. https://doi.org/10.1007/978-3-642-73309-3_14

[70] Del, G., et al. (1988) Structures, Correlations and Electromagnetic Interactions in the Living Matter. In: Froelich, H., Ed., Biological Coherence and Response to EXternal Stimuli, Springer Verlag, Berlin, 49-64. https://doi.org/10.1007/978-3-642-73309-3_3

[71] Janmey, P. (1995) Cell Membranes and the Cytoskeleton. In: Lipowsky, R. and Sackman, E., Eds., Handbook of Biological Physics, Vol. I, Elsevier Science, Amsterdam, 805-849. https://doi.org/10.1016/S1383-8121(06)80010-2

[72] Vincze, Gy. and Szasz, A. (2015) Reorganization of Actin Filaments and Microtubules by Outside Electric Field. Journal of Advances in Biology, 8, 1514-1518.

[73] Markovitch, O. and Agmon, N. (2007) Structure and Energetics of the Hydronium Hydration Shells. The Journal of Physical Chemistry A, 111, 2253-2256. https://doi.org/10.1021/jp068960g

[74] Szasz, A., van Noort, D., Scheller, A., et al. (1994) Water States in Living Systems. I. Structural Aspects. Physiological Chemistry and Physics, 26, 299-322. http://www.ncbi.nlm.nih.gov/pubmed/7700980 
[75] Agmon, N. (1995) The Grotthuss Mechanism. Chemical Physics Letters, 244, 456-462. https://doi.org/10.1016/0009-2614(95)00905-J

[76] Raff, M.C. (1992) Social Controls on Cell Survival and Death. Nature, 356, 397-400. https://doi.org/10.1038/356397a0

[77] Lanouette, W. (1992) Genius in the Shadows. Macmillan Publishing Co., New York, 350-361. 